Laser Chem.

Volume 1(1), October 1982, pp. 59-75

0278-6273/82/0101-0059\$6.50/0

(C) harwood academic publishers gmbh

Printed in the United States of America

\title{
Effect of Formation of van der Waals Complexes on Molecular Physical Properties
}

\author{
K. GODZIK*, T. R. HAYS†, W. E. HENKE†, H. L. SELZLE† and E. W. \\ SCHLAG† \\ Institute for Physical Chemistryt and Institute for Theoretical Chemistry* \\ Technical University of Munich 8046 Garching West Germany
}

S. H. LIN

Department of Chemistry Arizona State University Tempe, Arizona 85287 USA

(Received February 15, 1982; in final form March 2, 1982)

Experimental data for anthracene-argon van der Waals complexes are presented as a function of bound argon atoms. The results are interpreted by a new theoretical model which is based on the Hartree-Fock method and can explain molecular physical properties in the transition region from isolated gaseous molecules to the condensed phase.

\section{INTRODUCTION}

The major application of supersonic jet spectroscopy has been the investigation of ultracold, isolated molecules, van der Waals (vdW) complexes and clusters. ${ }^{1-10}$ The jet very effectively produces weakly bound species because of low internal temperatures attained and the relatively collisionfree flow which exists sufficiently far downstream from the nozzle. As a result a large variety of complexes can be studied, e.g., complexes between minor constituents and the carrier gas, or between the minor constituents themselves, or between different minor constituents with or without added carrier gas molecules, or between carrier gas molecules themselves. An important aspect is that the size of the complex can easily be varied by 
changing the experimental conditions. In this way it is possible to change stepwise from the gaseous isolated molecule to a larger and larger complex which finally closely resembles the condensed state. One has thus a unique method to study the transition from the microscopic to the macroscopic properties of matter. This method will certainly reveal many new facets about intermolecular interactions heretofore unstudiable. In the case of a guest molecule imbedded in host molecules one has the further advantage that the physical properties of the complex can be monitored by probing the guest molecule. Spectral shifts, linewidths, fluorescence lifetimes or ionization potentials of the guest molecule can be used, for example, to investigate the cluster properties. Along with these advances in experimental technique it was felt that a theoretical description is needed which is able to explain the photophysical properties in the transition region between microscopic and macroscopic behavior.

The purpose of this paper is two-fold. One is to report some recent experimental results on the photophysical properties of $\mathrm{vdW}$ complexes and the other is to present a theoretical treatment of the effect of formation of vdW complexes on these properties.

From studies of complexes $\mathrm{MA}_{n}$ (where $\mathrm{M}$ represents an aromatic molecule and $\mathrm{A}$ an inert-gas atom) with a low number of bound inert-gas atoms, direct information on nearest-neighbor interactions should be accessible, whilst as the experimental conditions are modified to increase the number of bound atoms, one may leave the regime characteristic of isolated gaseous molecules and approach the conditions more closely associated with inter-gas matrix studies.

\section{EXPERIMENTAL}

The system used and the experimental procedure were identical to that described earlier. ${ }^{6,11}$ Briefly, the ultraviolet radiation used for the fluorescence excitation was generated by mixing the infrared fundamental of a NdYAG laser (Quanta Ray DCR-1A) with the tunable laser radiation of the YAG-pumped dye laser (Quanta Ray PDL-1) using coumarin 540A (Lambda Physics). The anthracene used was heated in a home-built heated cell and the vapor introduced into the argon carrier gas stream. The fluorescence signal measured was normalized to the laser intensity for each shot, and ten to fifty such measurements were then averaged for each spectral point.

The fluorescence excitation spectrum of anthracene with argon carrier 
gas is shown in Figure 1 for four different stagnation pressures. Note that the intensity scale is about five times more sensitive for the complex peaks to the red of the $0-0$ transition. The intensities of the complex peaks are given in Table I. Unprimed peaks represent the stronger peaks in the series and primed ones the weak shoulders at lower pressures. These are not

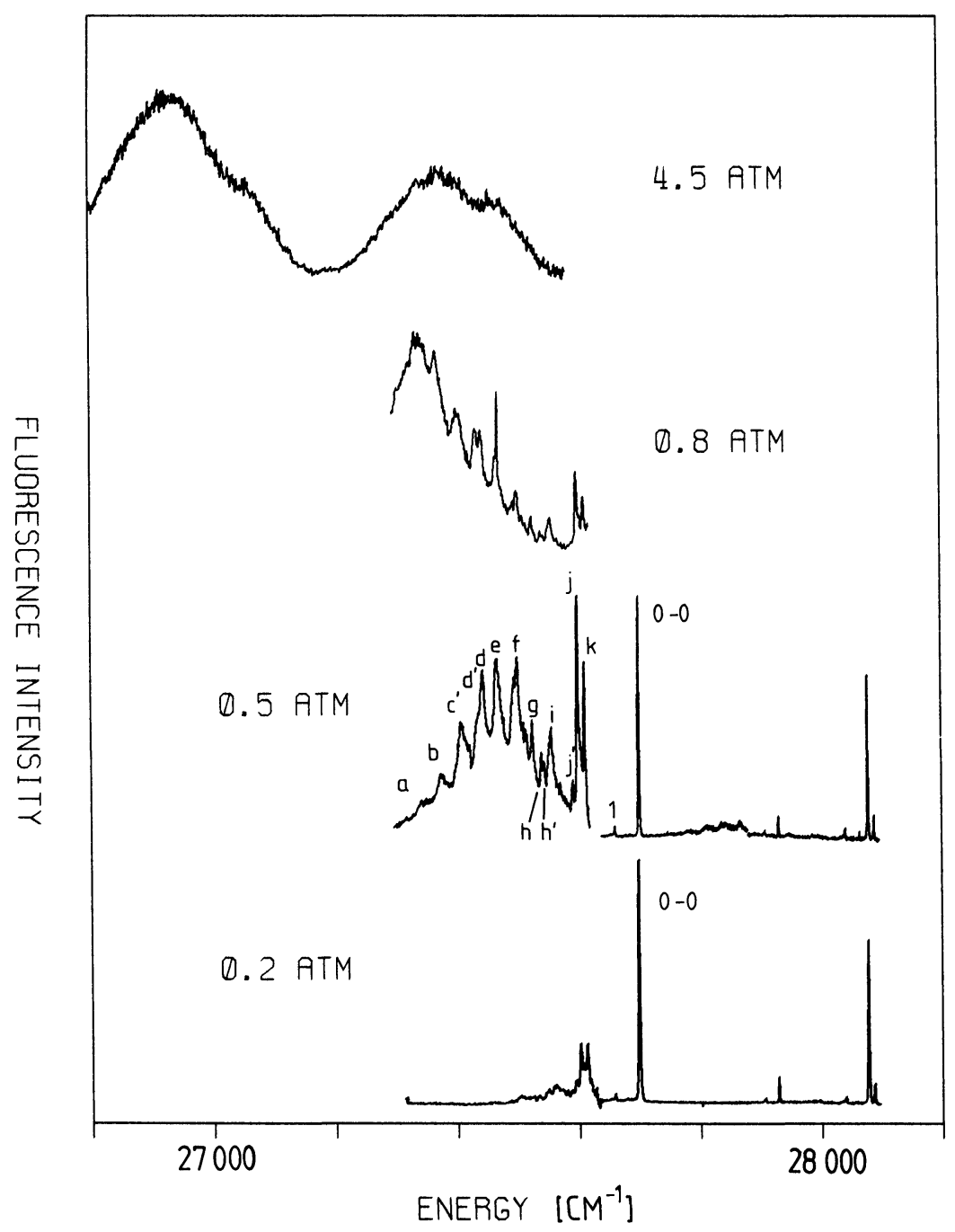

FIGURE 1 Fluorescence excitation spectrum of anthracene seeded in argon at different argon pressures. 
TABLE I

Argon pressure-dependence of complex peak intensities

\begin{tabular}{lccccccccc}
\hline Peak & $\begin{array}{c}-\left(v-v_{o o}\right) \\
\left(\mathrm{cm}^{-1}\right)\end{array}$ & $0.2 \mathrm{~atm}$ & $0.5 \mathrm{~atm}$ & $0.8 \mathrm{~atm}$ & slope & $n_{1 / 2}$ & $n_{1}$ & $n_{2}$ & $n_{s p}$ \\
\hline $0-0$ & 0 & 0 & 0 & 0 & 0 & 0 & 0 & 0 & 0 \\
1 & 32 & 1.62 & 1.39 & - & 0.6 & 1 & 1 & 0 & 1 \\
2 & 65 & 1.54 & 1.09 & - & 1.1 & 2 & 1 & 1 & 2 \\
$\mathrm{k}$ & 87 & 1.32 & 0.84 & 0.60 & 1.2 & 2 & 1 & 1 & 2 \\
$\mathrm{j}$ & 97 & 1.32 & 0.69 & 0.52 & 1.3 & 3 & 1 & 1 & 3 \\
$\mathrm{j}^{\prime}$ & 106 & 1.98 & 1.64 & - & 0.8 & 2 & 1 & 0 & 3 \\
$\mathbf{i}$ & 137 & 2.02 & 1.28 & 0.85 & 1.9 & 4 & 2 & 1 & 4 \\
$\mathrm{~h}^{\prime}$ & 148 & 2.32 & 1.76 & - & 1.4 & 3 & 1 & 1 & 4 \\
$\mathrm{~h}$ & 152 & 2.32 & 1.59 & 1.19 & 1.9 & 4 & 2 & 1 & 5 \\
$\mathrm{~g}$ & 166 & 2.42 & 1.34 & 0.94 & 2.5 & 5 & 3 & 1 & 5 \\
$\mathrm{f}$ & 190 & 2.32 & 1.09 & 0.76 & 2.6 & 5 & 3 & 1 & 6 \\
$\mathrm{f}^{\prime}$ & 193 & - & 1.64 & 0.96 & 3.4 & 7 & 3 & 2 & 6 \\
$\mathrm{e}$ & 220 & - & 1.16 & 0.62 & 2.7 & 5 & 3 & 1 & 7 \\
$\mathrm{~d}$ & 242 & - & 1.20 & 0.54 & 3.3 & 7 & 3 & 2 & 8 \\
$\mathrm{~d}^{\prime}$ & 249 & - & 1.76 & 0.60 & 5.8 & 11 & 6 & 3 & 8 \\
$\mathrm{c}^{\prime}$ & 275 & - & 1.34 & 0.70 & 3.2 & 6 & 3 & 2 & 9 \\
$\mathrm{~b}$ & 305 & - & 1.76 & 0.60 & 5.8 & 11 & 6 & 3 & 10 \\
$\mathrm{a}$ & 335 & - & 2.24 & 0.74 & 7.5 & 15 & 7 & 4 & 11 \\
\hline
\end{tabular}

equivalent to the series with arabic and roman numerals for tetracene, ${ }^{4}$ since there one sees marked pressure dependence differences which strongly indicate different numbers of bound argons. The intensities of the peaks are all scaled to the $0-0$, i.e.,

$$
I_{\text {rel }}=I / I_{0-0}
$$

To see the pressure dependence of the peaks, we assumed a relationship

$$
I_{r e l}^{i}(n)=A_{i}\left(P^{n}\right)^{\alpha}
$$

where $\mathrm{n}$ is the number of argons bound, $I_{\text {rel }}$ the relative intensity of the peak, $i$ the individual peak, $A_{i}$ a pressure-independent intensity factor which accounts for differences in absorption cross-section, fluorescence quantum yield, the thermodynamic and kinetic probability of the complex being formed, etc., $P$ the stagnation pressure of the carrier gas, and $\alpha$ the overall pressure dependence of the step $\mathrm{C}_{14} \mathrm{H}_{10}+n \mathrm{Ar} \rightarrow \mathrm{C}_{14} \mathrm{H}_{10} \mathrm{Ar}_{n}$. Reexpressing (2) gives a more usable form 


$$
\log \left(I_{\text {rel }}^{i}(n)\right)=\log A_{i}+\alpha n \log P
$$

so that a plot of $\log I$ vs. $\log P$ should give a slope of $\alpha$ n. This is the slope listed in Table I.

A major difficulty that remains is that both $\alpha$ and $n$ are unknown. Thus, one needs to assume a value for one and then see how the experimental results fit the pattern. In Table I, fits have been attempted using $\alpha=\frac{1}{2}$, 1 , and 2 and the resulting $n_{\alpha}$ calculated. These $\alpha$ values were chosen for various reasons: $\alpha=2$, since that was the best fit value seen in earlier work on other molecules (4); $\alpha=1$, which assumes that complex formation occurs from two-particle collisions; and $\alpha=\frac{1}{2}$, used because the slopes, at least for lower values of $n$, seemed to cluster at roughly this spacing. In addition, a fourth set of values, $n_{s p}$, was based purely on the spacing of the more intense complex peaks, with the shoulders and other weak peaks then to fit in entirely on the basis of their spectral positions. This latter series gave $\alpha$ values with a mean and standard deviation of $0.48 \pm$ 0.12 . Ignoring the primed peaks gives mean values of $0.49 \pm 0.11$, not significantly different.

Comparing the various predictions, $\alpha=\frac{1}{2}$ gives the best apparent fit for the three sets of $n_{\alpha}$. The prediction of six different $\mathrm{C}_{14} \mathrm{H}_{10} \mathrm{Ar}$ complexes for $\alpha=1$ is quite unreasonable, while $\alpha=2$ predicts two "complexes" which have no argons at all and nine with one argon. Also, the $n_{1 / 2}$ values are in good agreement with the $n_{s p}$ values at least up to $n_{s p}=5$. However, the $\alpha=\frac{1}{2}$ relationship is difficult to explain in terms of a reasonable physical model. This may in part be due to the experimental technique. The relationship uses the stagnation gas pressure, which may not be valid for the pulsed nozzle arrangement employed. Instead, a pressure gradient might be established after the nozzle opens, which would alter the actual pressure in the cooling region of the jet. Also, there is a broad background underlying the resolved complex peaks, which leads to some error in determining the peak intensities. Both of these could give systematic errors in the slopes. In addition, there is undoubtedly an error resulting from the small number of points (two or three) for each plot. While this could easily cause the fluctuations in the slopes, it is not an explanation for such small slopes, with the steepest not close to one for the two better fits of the data. In short, the results from pressure dependence and spectral shifts seem consistent, up to about $n=5$, but the value of $\alpha=\frac{1}{2}$ is difficult to explain mechanistically.

Comparing the $n$ values to the spectral shifts, we see a series for $n=$ 
$0,1,2$, and 3 with spacings of about $32 \mathrm{~cm}^{-1}$ (peaks $0-0,1,2$, and $\mathrm{j}$ ). Since anthracene has three rings, this could represent the binding of 0 to 3 argons, no two of which are on the same ring. This series seems to quit at $n=3$. It is possible to find other series, such as $j^{\prime}, i, g, f^{\prime}$ or $f, e, d^{\prime}$, $c, b, a$, both with spacings of about $30 \mathrm{~cm}^{-1}$, but these are always a mixture of the stronger peaks and the weak peaks or shoulders. Thus, they are more likely coincidences rather than true series.

\section{THEORY}

To study the effect of formation of van der Waals complexes on the spectral shift and ionization potential, we shall employ the Hartree-Fock molecular orbital theory as a preliminary attempt to treat this problem. The effect of electron correlation will be considered in future studies.

The ground state of van der Waals complexes $\mathrm{MA}_{n}$ will be assumed to be closed-shell. Since the interaction between $M$ and $A$ is weak, the molecular orbitals, $\phi_{i}$ can still be classified as those belonging to $M, \phi_{i}(M)$, and those belonging to A's, $\phi_{i}(\mathrm{~A})$. According to the H-F theory, the total energy $E$ can be expressed as ${ }^{12}$

$$
E=2 \sum_{i} \varepsilon_{i}-\sum_{i} \sum_{j}\left(2 J_{i j}-K_{i j}\right)
$$

where $\varepsilon_{i}$ is the orbital energy, $J_{i j}$, the Coulomb integral and $K_{i j}$, the exchange integral.

$$
\begin{aligned}
\varepsilon_{i} & =<\phi_{i}\left|H_{\mu}\right| \phi_{i}>+\sum_{j}\left(2 J_{i j}-K_{i j}\right) \\
J_{i j} & =<\phi_{i} \phi_{j}\left|\frac{e^{2}}{r_{\mu \nu}}\right| \phi_{i} \phi_{j}>
\end{aligned}
$$

and

$$
K_{i j}=<\phi_{i} \phi_{j}\left|\frac{e^{2}}{r_{\mu \nu}}\right| \phi_{j} \phi_{i}>
$$

$\varepsilon_{i}$ and $\phi_{i}$ are determined by the H-F equation

$$
\left[\hat{H}_{\mu}+\sum_{j}\left(2 J_{j}-K_{j}\right)\right] \phi_{i}=\varepsilon_{i} \phi_{i}
$$


The singlet and triplet excitation energies for the transition from $\phi_{i}$ to $\phi_{a}$ are given by

$$
E\left(S_{i \rightarrow a}\right)-E\left(S_{0}\right)=\varepsilon_{a}-\varepsilon_{i}-\left(J_{i a}-K_{i a}\right)+K_{i a}
$$

and

$$
E\left(T_{i \rightarrow a}\right)-E\left(S_{0}\right)=\varepsilon_{a}-\varepsilon_{i}-\left(J_{i a}-K_{i a}\right)-K_{i a}
$$

respectively. The ionization energy for the electron to be removed from the $\phi_{i}$ orbital is given by

$$
I_{i}=-\varepsilon_{i}
$$

Now we are ready to treat the effect of formation of van der Waals complexes on the spectroscopic properties. In the lowest order of approximation, we may expect this effect to originate from $\varepsilon_{i}$ and/or $\varepsilon_{a}$. Notice that for example

$$
\varepsilon_{i}(\mathbf{M})=<\phi_{i}\left|\hat{H}_{\mu}\right| \phi_{i}>+\sum_{j}\left(2 J_{j i}-K_{j i}\right)
$$

where $\varepsilon_{i}(\mathrm{M})$ denotes the orbital energy for the $i$-th $\mathrm{MO}$ belonging to $\mathrm{M}$. The Hamiltonian $\hat{H}_{\mu}$ due to the electron moving around the bare nuclei of $\mathrm{MA}_{n}$ can be separated as

$$
\begin{aligned}
<\phi_{i}(\mathrm{M})\left|\hat{H}_{\mu}\right| \phi_{i}(\mathrm{M})>=<\phi_{i}(\mathrm{M}) \mid & \hat{H}_{\mu}^{0} \mid \phi_{i}(\mathrm{M})> \\
& +<\phi_{i}(\mathrm{M})\left|V_{A}\right| \phi_{i}(\mathrm{M})>
\end{aligned}
$$

where $\hat{H}_{\mu}^{0}$ is the one electron Hamiltonian for the unperturbed molecule and $V_{A}$ is the potential function for the interaction between the electron and the nuclei of A's. Similarly, the two-electron terms in Eq. (3-9) can be separated as

$$
\sum_{j}\left(2 J_{j i}-K_{j i}\right)=\sum_{j(M)}\left(2 J_{j i}-K_{j i}\right)+\sum_{j(A)}\left(2 J_{j i}-K_{j i}\right)
$$

where the summations over $j(M)$ and $j(A)$ refer to the summations over the MO's of M and the MO's of A's respectively.

It should be noted that although $\phi_{i}(\mathrm{M})$ is essentially the $\mathrm{MO}$ of $\mathrm{M}$, due 
to the interaction between $M$ and A's it is not the pure MO $\phi_{i}^{0}(\mathrm{M})$ of $\mathrm{M}$; however the difference $\phi_{i}(\mathrm{M})$ and $\phi_{i}^{0}(\mathrm{M})$ in this case is small and may be treated by the perturbation method. Using Eqs. (3-10) and (3-11) we can rewrite Eq. (3-9) as

$$
\varepsilon_{i}(\mathbf{M})=\varepsilon_{i}^{0}(\mathbf{M})+\Delta \varepsilon_{i}
$$

where $\Delta \varepsilon_{i}$ represents the change of the orbital energy due to the vdW complex formation, i.e.,

$$
\Delta \varepsilon_{i}=<\phi_{i}(\mathrm{M})\left|V_{A}\right| \phi_{i}(\mathrm{M})>+\sum_{j(A)}\left(2 J_{j i}-K_{j i}\right)
$$

We now treat the effect of the number of bound A's in $\mathrm{MA}_{n}$ on the spectral shift due to the vdW complex formation. From Eqs. (3-6) and (37) we can see that the difference between the singlet and the triplet excitation energies is due to the exchange integral $K_{i a}$ alone. Thus for the case in which the differences $\phi_{i}(\mathrm{M})-\phi_{i}^{0}(\mathrm{M})$ and $\phi_{a}(\mathrm{M})-\phi_{a}^{0}(\mathrm{M})$ are negligible we may expect that the spectral shifts for $S_{0} \rightarrow S_{i \rightarrow a}$ and $S_{0} \rightarrow$ $T_{i \rightarrow a}$ are approximately the same. In this case, the spectral shift can be expressed as

$$
\Delta E(n)_{i \rightarrow a}=\Delta \varepsilon_{a}(n)-\Delta \varepsilon_{i}(n)
$$

If $n$ in $\mathrm{MA}_{n}$ is small, the adsorbed A's may have various equivalent positions on the $M$ molecule; these equivalent positions will be classified as $\alpha, \beta \ldots$ etc. It follows that

$$
\begin{aligned}
\Delta E(n)_{i \rightarrow a}=n_{a}\left[\Delta \varepsilon_{a}(\alpha)-\Delta \varepsilon_{i}(\alpha)\right] & \\
& +n_{\beta}\left[\Delta \varepsilon_{a}(\beta)-\Delta \varepsilon_{i}(\beta)\right]+\ldots
\end{aligned}
$$

where $n=n_{\alpha}+n_{\beta}+\ldots$ and for example

$$
\Delta \varepsilon_{i}(\alpha)=<\phi_{i}(\mathrm{M})\left|V_{A}(\alpha)\right| \phi_{i}(\mathrm{M})>+\sum_{j(A \alpha)}\left(2 J_{j i}-K_{j i}\right)
$$

The determination of the distribution $n_{\alpha}, n_{\beta} \ldots$ depends on the relative stabilities of the types of adsorbed A's classified according to $\alpha, \beta, \ldots$ The stability problem of $\mathrm{MA}_{n}$ can in principle be also treated by the H-F theory; 
however, electron correlation may be quite important in this problem. From Eq. (3-15) we can see that the slopes of the straight lines $\Delta E(n)_{i \rightarrow a}$ vs $n_{\alpha}$ and $\Delta E(n)_{i \rightarrow a}$ vs $n_{\beta}$ are different; this is borne out by recent experiment results. $^{5}$

Next we consider the case of large $n$ values; in this case except for a small number of adsorbed A's in the first several layers of $\mathrm{MA}_{n}$, the remaining adsorbed A's in $\mathrm{MA}_{n}$ can be described by a distribution function $\mathrm{g}_{i a}(\mathbf{r})$. That is,

$$
\Delta E(n)_{i \rightarrow a}=\Delta E_{d}(n)_{i \rightarrow a}+\Delta E_{c}(n)_{i \rightarrow a}
$$

where

$$
\begin{aligned}
\Delta E_{d}(n)_{i \rightarrow a}=n_{\alpha}\left[\Delta \bar{\varepsilon}_{\alpha}(\alpha)-\Delta \bar{\varepsilon}_{i}(\alpha)\right] & \\
& +n_{\beta}\left[\Delta \bar{\varepsilon}_{a}(\beta)-\Delta \bar{\varepsilon}_{i}(\beta)\right]+\ldots
\end{aligned}
$$

and

$$
\Delta E_{c}(n)_{i \rightarrow a}=\rho \int g_{i a}(\mathbf{r}) d \mathbf{r}\left[\Delta \bar{\varepsilon}_{a}(r)-\Delta \bar{\varepsilon}_{i}(r)\right]
$$

If the distribution of adsorbed A's can be assumed to be spherically symmetric around $\mathrm{M}$, then $\mathrm{Eq}$. (3-19A) reduces to

$$
\Delta E_{c}(n)_{i \rightarrow a}=\rho \int_{r_{o}}^{R} g_{i a}(r) d r\left[\Delta \bar{\varepsilon}_{a}(r)-\Delta \bar{\varepsilon}_{i}(r)\right]
$$

Here for simplicity it is assumed that besides $n_{\alpha}, n_{\beta}, \ldots$ the adsorbed A's are continuously distributed between $r_{0}$ and $R$. Notice that $\rho$ in Eq. (3.19) denotes the macroscopic density (in number of A's per unit volume) of the A crystal.

From Eqs. (3-18) and (3-19) we can see that the measurement of the spectral shift or ionization potential as a function of cluster size $n$ in $\mathbf{M A}_{n}$ can be used as a probe to study the molecular dynamics of clusters. ${ }^{2-7}$ Investigations concerning the formation and properties of small clusters comprise an active area of research both experimentally and theoretically. ${ }^{8-10}$ 
To determine $\Delta E_{c}(n)_{i \rightarrow a}$ it is necessary to know $g_{i a}(r), \Delta \bar{\varepsilon}_{a}(r)$ and $\Delta \bar{\varepsilon}_{i}(r)$. For simplicity we shall assume a random distribution for $\mathrm{g}_{i a}(r)$, i.e., $\mathrm{g}_{i a}(r)$ $=4 \pi r^{2}$. From Eq. (3-13) or Eq. (3-16), we can see that $\Delta \varepsilon_{i}$ represents the interaction energy between the electron of $\mathrm{M}$ in the $i$-th MO $\phi_{i}(\mathrm{M})$ and A's; while the terms like $<\phi_{i}(\mathrm{M})\left|V_{A}\right| \phi_{i}(\mathrm{M})>+2 \sum_{j(A)} J_{j i}$ represent the London type dispersion force, the terms like $\sum_{j} K_{j i}$ represent the short range force. Thus we shall assume $\Delta \bar{\varepsilon}_{i}(r)$ to take the following form.

$$
\Delta \bar{\varepsilon}_{i}(r)=\frac{\lambda_{i}}{r^{6}}+\frac{\lambda_{i}{ }^{\prime}}{r^{12}}
$$

It should be noted that for the case in which the size of $\mathrm{MA}_{n}$ becomes macroscopic, the influence (or the interaction) among adsorbed $A$ 's on their atomic or molecular orbitals involved in $\Delta \varepsilon_{i}(n)$ and $\Delta \varepsilon_{a}(n)$ may be quite significant; this effect can in part be taken into consideration by introducing the dielectric constant of the solvent A lattice.

Using these results for $\mathrm{g}_{i a}(r), \Delta \bar{\varepsilon}_{a}(r)$ and $\Delta \bar{\varepsilon}_{i}(r)$, we obtain

$$
\Delta E_{c}(n)_{i \rightarrow a}=\Delta E_{c}(\infty)_{i \rightarrow a}+4 \pi \rho\left[\frac{\left(\lambda_{i}-\lambda_{a}\right)}{3 R^{3}}+\frac{\left(\lambda_{i}{ }^{\prime}-\lambda_{a}{ }^{\prime}\right)}{9 R^{9}}\right]
$$

where $\Delta E_{c}(\infty)_{i \rightarrow a}$ represents the limiting value of $\Delta E_{c}(n)_{i \rightarrow a}$ (i.e., the macroscopic value of $\left.\Delta E_{c}(n)_{i \rightarrow a}\right)$,

$$
\Delta E_{c}(\infty)_{i \rightarrow a}=-4 \pi \rho\left[\frac{\left(\lambda_{i}-\lambda_{a}\right)}{3 r_{0}^{3}}+\frac{\left(\lambda_{i}^{\prime}-\lambda_{a}^{\prime}\right)}{9 r_{0}^{9}}\right]
$$

If $n_{e}$ represents the number of A's distributed between $R$ and $r_{0}$, i.e., $n_{e}$ $=n-\left(n_{\alpha}+n_{\beta}+\ldots\right)$, then $R$ can be related to $n_{e}$ or $n$ by

$$
R^{3}=r_{0}^{3}+\left(\frac{3 n_{e}}{4 \pi \rho}\right)
$$


In other words, the second term in Eq. (3-21) describes how the spectral shift approaches the limiting macroscopic value as $n$ increases.

It should be noted that for large $n$ values, $r_{0}$ in Eq. (3-23) may be negligible compared with the term $\left(3 n_{e} / 4 \pi \rho\right)$. In this case, Eq. (3-21) becomes

$$
\begin{aligned}
\Delta E_{c}(n)_{i \rightarrow a}=\Delta E_{c}(\infty)_{i \rightarrow a}+\frac{16 \pi^{2} \rho^{2}\left(\lambda_{i}-\right.}{\left.\lambda_{a}\right)} & \\
9 n_{e} & +\frac{256 \pi^{4} \rho^{4}\left(\lambda_{i}{ }^{\prime}-\lambda_{a}{ }^{\prime}\right)}{243 n_{e}{ }^{3}}
\end{aligned}
$$

and we will also have $n_{e} \doteqdot n$.

It should be noted that in the above discussion of the size dependence of the spectral shift $\mathrm{MA}_{n}$, for simplicity a random distribution function has been employed for the radial distribution function $g_{i a}(r)$. For a given interaction potential function the radial distribution function can be determined not only for an extended system but also for small clusters. ${ }^{13}$

The effect of the number of bound $\mathrm{A}$ 's in $\mathrm{MA}_{n}$ on the ionization potential due to the vdW complex formation can be treated similarly by using Eq. (3-8) so its treatment will not be given here. It should be noted that, in this case, as $n$ in $\mathrm{MA}_{n}$ approaches the macroscopic value, the mechanism of photoionization may not be the same as that for small $n$.

\section{DISCUSSION}

In Section 3, we have presented a theory of the effect of vdW complex formation on spectroscopic properties. In this section we shall apply the theory to our experimental results and the experimental results of others.

Beck et al. ${ }^{2}$ have measured the fluorescence excitation spectra of benzene and benzene-helium vdW complexes. They obtained the spectral shifts as follows $\Delta v(\mathrm{MA})=-69.11 \pm 0.60$ and $\Delta \nu\left(\mathrm{MA}_{2}\right)=-138.29 \pm 0.65$ $\mathrm{GHz}$; that is, the linear relation between the spectral shifts and $n$ of $\mathbf{M A}_{n}$ is obtained in this case. This is because the two He atoms adsorbed occupy equivalent positions on the benzene molecule. For iodine van der Waals complexes with neon and helium $\mathrm{I}_{2} \mathrm{He}_{a} \mathrm{Ne}_{b}$ the spectral frequency depends linearly on the number of bound helium $(a)$ and neon $(b)$ atoms for $b<$ 
7 (Ref. 3), $v-v_{o}=3.67 a+6.25 b\left(\mathrm{~cm}^{-1}\right)$. For $\mathrm{I}_{2} \mathrm{Ne}_{7}$ this band shift rule breaks down which indicates that there are six equivalent sites for neon to be bound to iodine.

Similar spectral shifts of $\mathrm{MA}_{n}$ are shown in Figures 2 and 3 for anthracene and tetracene. Again for small $n$ values, either linear or bi-linear relations have been observed as expected from the theory. As mentioned in Section 2 there exist more spectral shift series in tetracene than in anthracene. One possible cause is the smaller size of anthracene, since the complexes argons would be crowded around a smaller core and thus might interact more strongly with each other. Since our model, or any model which assumes equal spacings for equivalent sites, assumes interactions between argons to be negligible, this could be a contributing factor. However, the consistency of the tetracene results up to at least $n=6$ (Ref. 5) would seem to indicate that the series with anthracene should go beyond $n=3$.

A second possibility is that the difference $\phi_{i}(\mathrm{M})-\phi_{i}^{0}(\mathrm{M})$ is not negligible. In the series benzene, naphthalene, anthracene, etc., the $S_{1}$ and $S_{2}$ are symmetry-forbidden and a symmetry-allowed state. For benzene and naphthalene, the $S_{1}$ is the forbidden state while the $S_{2}$ is allowed. Starting with anthracene, however, the $S_{1}$ is the allowed state. Buried under the $S_{1}$ somewhere, though, is still the symmetry-forbidden state. For anthracene, these two states should be much closer together than for tetracene. If this is indeed the case, any slight perturbation could cause a large change in the mixing of the atomic orbitals and thus in the character of the $S_{1}$ and $S_{2}$ states. This would render some of the theoretical asumptions invalid and would render the concept of equivalent sites less important if not meaningless, since the "equivalent" sites would be altered depending on where an additional argon might bind. Assuming that the relative lowering of the allowed state compared to the forbidden state continues, then the perturbation of the MO's should be much less for tetracene than for anthracene. This expectation of a more complex pattern in anthracene versus a much more regular pattern in tetracene seems to be supported by the experimental results.

It should be noted that the spectral shift of anthracene in a polymethylmethacrylate matrix is $1250 \mathrm{~cm}^{-1},{ }^{14}$ while the spectral shift of tetracene in the naphthalene matrix is $2100 \mathrm{~cm}^{-1} .{ }^{15}$ We have measured the spectral shift of the anthracene-argon complex with approximately $n=100$ to be $\delta v(n \approx 100)=760 \mathrm{~cm}^{-1}$. The value of $n$ could only be estimated from the pressure dependence and the spectral shift rule. Further mass spectrometric experiments are underway to clarify this point. 


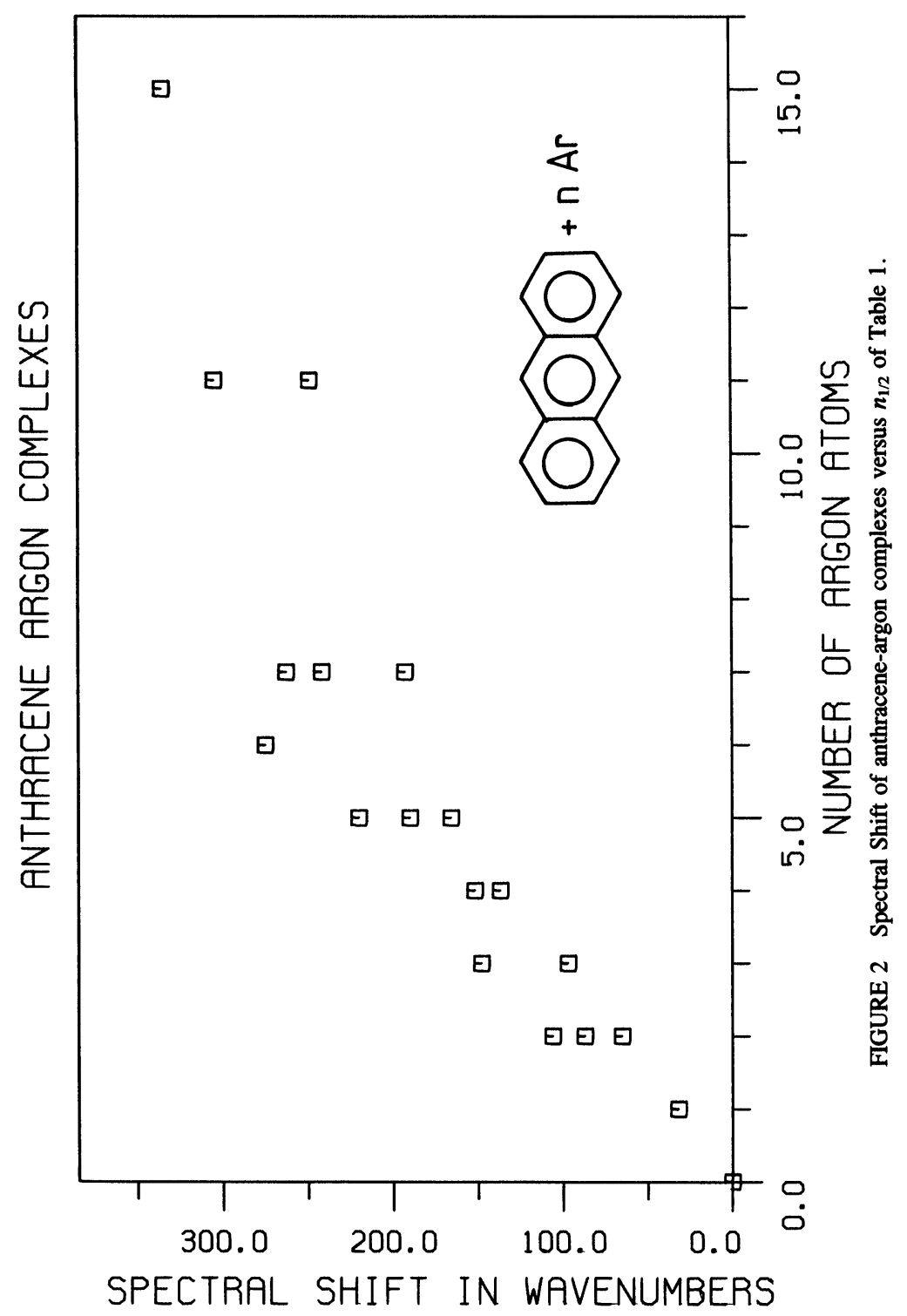




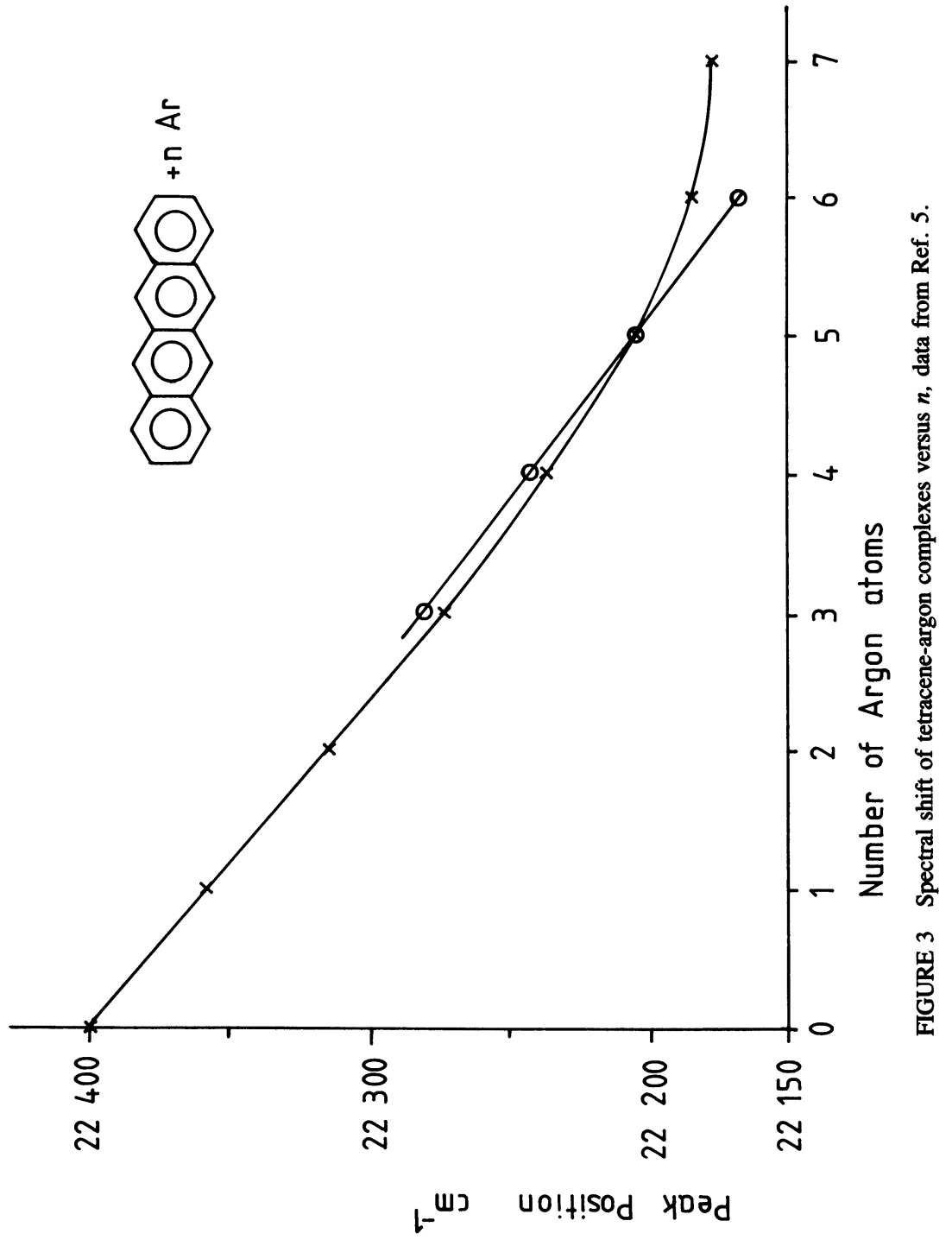


The ionization potentials of benzene and benzene-argon complex have been reported ${ }^{7}$; they are $I$ (benzene) $=9.240 \mathrm{eV}$ and $I$ (benzene-argon) $=$ $9.2188 \mathrm{eV}$, respectively. That is the shift of the ionization potential is $-171 \mathrm{~cm}^{-1}$, which should be compared with that in the argon matrix of $-6000 \mathrm{~cm}^{-1}(0.73 \mathrm{eV}) .^{16}$

The ionization potential of $\left(\mathrm{H}_{2} \mathrm{~S}\right)_{n}{ }^{+}$as a function of $n$ has been reported. ${ }^{17}$ Although the theory for the shift of ionization potentials should be somewhat different from that presented in Section 3 because of the resonance interaction, we have nevertheless shown the results in Figure 4. As can be seen from this figure, the shift varies with $1 / n^{2}$. The SCF-CI calculation, exciton theory, free electron theory etc., have been proposed to interpret the size effect. ${ }^{18}$

Let us now compare our treatment of the spectral shift for $\mathrm{MA}_{n}$ with the conventional theory of solvent effects on molecular electronic spectra. ${ }^{19}$ In this treatment, the basis set of the total system (i.e., a solute molecule plus solvent molecules) is written as a product

$$
\psi_{m n}=\psi_{m} \text { (solute) } \psi_{n} \text { (solvent). }
$$

The interaction between the solute molecule and solvent molecules is chosen to be of the dipole-dipole interaction, and the perturbation method is then employed to calculate the solvent effect on the electronic energy. Further simplifications are accomplished by using Onsager's dielectric model. This conventional theory of spectral shift due to the solvent effect can only provide limiting results $\Delta E(\infty)_{i \rightarrow a}$ and cannot treat the cluster size dependence of the spectral shift.

From Eq. (3-17) we can see that the spectral shift (or ionization potential) for large clusters can be separated into the discrete part $\Delta E_{d}(n)_{i \rightarrow a}$ and the continuous part $\Delta E_{c}(n)_{i \rightarrow a}$. For a macroscopic size of $\mathrm{MA}_{n}$, the discrete contribution $\Delta E_{d}(n)_{i \rightarrow a}$ is usually less than $10 \%$ of the continuous contribution $\Delta E_{c}(n)_{i \rightarrow a}$; that is the reason that the conventional theory of solvent effects on molecular electronic spectra applies.

In concluding this paper, it should be noted that for $\mathrm{MA}_{n}$, the spectral shift for $i \rightarrow a$ and the shift of ionization potential for the ionization from the $i$-th MO are given by $\Delta E(n)_{i \rightarrow a}=\Delta \varepsilon(n)-\Delta \varepsilon_{i}(n)$ and $\Delta I(n)_{i}=-$ $\Delta \varepsilon_{i}(n)$, respectively. Thus from the combined determination of $\Delta E(n)_{i \rightarrow a}$ and $\Delta V(n)_{i}$ we can determine both $\Delta E_{i}(n)$ and $\Delta \varepsilon_{a}(n)$. These quantities are particularly important for $n=1$, i.e., for MA, because they represent the interaction energy between an electron in a particular MO of $M$ and $A$. 
K. GODZIK ET AL.

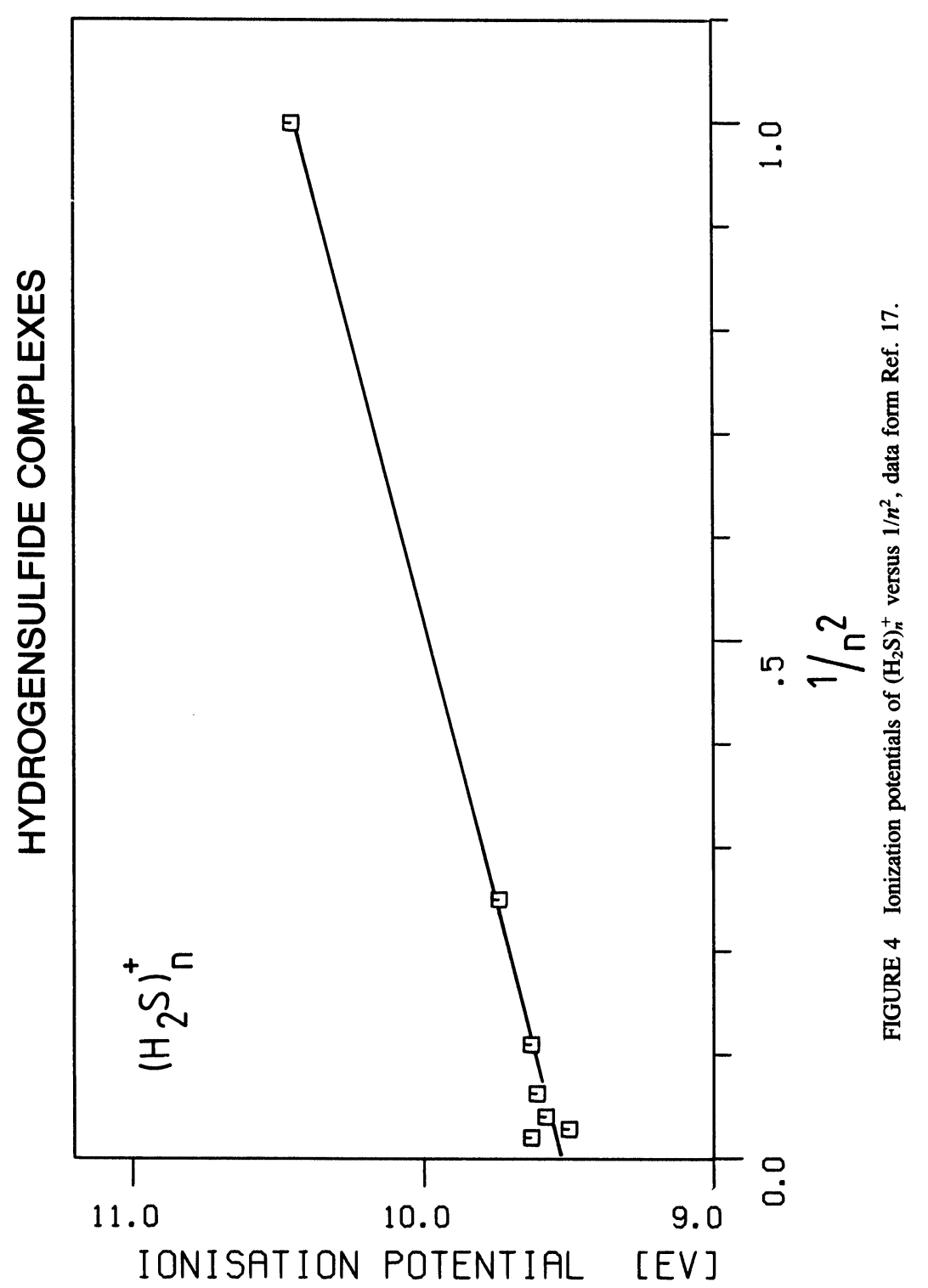


The quantities like $\Delta \varepsilon_{i}(n)$ and $\Delta \varepsilon_{a}(n)$ can in principle be evaluated by the ab initio calculation by either the H-F method or the SCF LCAO method. However, in this paper we have only used them to derive the theoretical relations for correlating and interpreting the experimental results.

One of us (S. H. Lin) acknowledges an award from the Alexander von Humboldt foundation which permitted a stay during which this work was initiated.

\section{References}

1. D. H. Levy, L. Wharton and R. E. Smalley, Acc. Chem Res. 10, (1977) 134.

2. S. Beck, M. G. Liverman, D. L. Monts and R. E. Smalley, J. Chem. Phys. 70, (1979) 232.

3. J. E. Kenny, K. E. Johnson, W. Sharfin and D. H. Levy, J. Chem. Phys. 72, (1980) 1109.

4. A. Amirav, U. Even and J. Jortner, J. Chem. Phys. 75 (1981) 2489; Chem. Phys. Letters 67 (1979) 9; Chem. Phys. 51, (1980) 31.

5. I. Raitt, A. M. Griffiths and P. A. Freedman, Chem. Phys. Letters 80, (1981) 225.

6. T. R. Hays, W. Henke, H. L. Selzle and E. W. Schlag, Chem. Phys. Letters 77, (1981) 19; Z. Naturforschung 35a (1980) 1429.

7. K. H. Fung, W. E. Henke, T. R. Hays, H. L. Selzle and E. W. Schlag, J. Phys. Chem. 85, (1981) 3560 .

8. R. D. Etters, K. Flurchick, R. P. Pan and V. Chandrasekharan, J. Chem. Phys. 75, (1981) 929.

9. M. J. Vasile and F. A. Stevie, J. Chem. Phys. 75, (1981) 2399.

10. B. D. Kay, V. Hermann and A. W. Castleman, Jr., Chem. Phys. Letters 80, (1981) 469.

11. W. E. Henke, T. R. Hays, H. L. Selzle, E. W. Schlag and S. H. Lin, J. Chem. Phys. 76, (1982) 1327

12. C. C. J. Roothaan, Rev. Mod. Phys. 23, (1951) 69.

13. D. Frenkel and J. P. McTague, Ann. Rev. Phys. Chem. 31, (1980) 491.

14. L. M. Logan, I. H. Munro, D. F. Williams and F. R. Lipsett, in: Molecular Luminéscence, ed. E. C. Lim (Benjamin, New York, 1979) p. 773.

15. J. W. Sidman, J. Chem. Phys. 25, (1956) 122.

16. J. Jortner, in: Vacuum Ultraviolet Radiation Physics, ed. E. E. Koch, R. Haensel, and C. Kunz (Pergamon, Vieweg, 1974) p. 291.

17. E. A. Walters and N. C. Blais, J. Chem. Phys. 75, (1981) 4208.

18. A. Szabo, J. Lauglet and J. P. Malrieu, Chem. Phys. 13, (1976) 173.

19. S. Basu, Advances in Quantum Chemistry 1, (1964) 145. 